\title{
Fast method for force computations in electronic structure calculations
}

\section{Citation}

Choly, Nicholas, and Efthimios Kaxiras. 2003. "Fast Method for Force Computations in Electronic Structure Calculations.” Physical Review B 67 (15). https://doi.org/10.1103/physrevb.67.155101.

\section{Permanent link}

http://nrs.harvard.edu/urn-3:HUL.InstRepos:41384085

\section{Terms of Use}

This article was downloaded from Harvard University's DASH repository, and is made available under the terms and conditions applicable to Other Posted Material, as set forth at http:// nrs.harvard.edu/urn-3:HUL.InstRepos:dash.current.terms-of-use\#LAA

\section{Share Your Story}

The Harvard community has made this article openly available.

Please share how this access benefits you. Submit a story.

\section{Accessibility}




\title{
Fast method for force computations in electronic structure calculations
}

\author{
Nicholas Choly* and Efthimios Kaxiras \\ Department of Physics and Division of Engineering and Applied Sciences, \\ Harvard University, \\ Cambridge, MA 02138
}

(Dated: October 27, 2018)

\begin{abstract}
We present new efficient $(O(N \log N))$ methods for computing three quantities crucial to electronic structure calculations: the ionic potential, the electron-ion contribution to the Born-Oppenheimer forces, and the electron-ion contribution to the stress tensor. The present methods are applicable to calculations in which the electronic charge density is represented on a uniform grid in real space. They are particularly well-suited for metallic extended systems, where other $O(N)$ methodologies are not readily applicable. Based on a fast algorithm for determining the atomic structure factor, originally developed by Essmann et al. [1] for fast Ewald energy and force computation, the present methods involve approximations that can be systematically improved. The methods are tested on a representative metallic system (bulk $\mathrm{Al}$ ), and their ability to simultaneously achieve high accuracy and efficiency is demonstrated.
\end{abstract}

PACS numbers: 71.15.-m, 71.15.Dx

A wealth of efficient methods have recently been developed 2, 3, 4, 5, 6, 7, 8, 9, 10, 11, 12] for calculating electronic properties of an extended physical system that require an amount of computation that scales linearly with the size of the system $N$. This size can be defined to be the number of atoms or the number of valence electrons, or the volume of the system, all of which are linearly related for large condensed systems. In this article we present a quasi-linear-scaling $(O(N \log N))$ method for computing the ionic potential, the ionic forces, and the stress tensor in electronic structure calculations. Atomic forces are necessary for the calculation of many physical properties of a system, including the determination of the optimal structure and simulation at a finite temperature. Some linear-scaling methods achieve linear computational scaling for the computation of the energy but not for the forces on all of the ions $[8]$. Other linear-scaling methods achieve efficient force calculations by working with a basis of localized functions 6 , [9, 10], which are less efficient at representing delocalized electronic states found for example in metallic systems. The present method applies to calculations performed in a periodic parallelepiped supercell, not necessarily orthogonal, in which the electronic charge density $\rho(\mathbf{r})$ is represented on a uniform grid.

As a result of the Hellmann-Feynman [13, 14] theorem, the force on the $p$ th ion (within the Born-Oppenheimer approximation) is given by the sum of the partial derivatives of the ion-ion energy (the Ewald energy) and the electron-ion energy with respect to the atomic coordinates:

$$
\mathbf{F}_{p}=\mathbf{F}_{p}^{E \text { wald }}+\mathbf{F}_{p}^{e-i}=-\frac{\partial E^{\text {Ewald }}}{\partial \mathbf{t}_{p}}-\frac{\partial E^{e-i}}{\partial \mathbf{t}_{p}}
$$

\footnotetext{
${ }^{*}$ Electronic Address: choly@fas.harvard.edu
}

where

$$
E^{e-i} \equiv \int_{\text {cell }} \rho(\mathbf{r}) V^{i o n}(\mathbf{r}) d \mathbf{r}
$$

and the ionic potential is defined as:

$$
V^{i o n}(\mathbf{r})=\sum_{\mathbf{R}} \sum_{p=1}^{N_{a t}} V^{p s p}\left(\mathbf{r}-\mathbf{t}_{p}-\mathbf{R}\right)
$$

where here the $\mathbf{t}_{p}$ are the atomic positions within the unit cell; $V^{p s p}(r)$ is the pseudopotential representing the ions; and the outer sum over $\mathbf{R}$ is over all lattice translation vectors $\mathbf{R}=n_{1} \mathbf{a}_{1}+n_{2} \mathbf{a}_{2}+n_{3} \mathbf{a}_{3}$ for all integers $n_{i}$, where the $\mathbf{a}_{i}$ are the lattice vectors defining the unit cell. For simplicity of presentation, we will consider systems that involve only one type of pseudopotential, but the methods presented here generalize readily to systems with multiple pseudopotentials. Furthermore, non-local pseudopotentials can be split into a short-ranged nonlocal part and a long-ranged local part; only the longranged local part of $V^{p s p}$ will be considered here. It should be noted that the present methods make only part of the electronic structure calculation efficient, and in the context of the Kohn-Sham method, the overall electronic structure calculation will still scale as $N^{3}$ due to the need to orthogonalize $N$ wavefunctions or to diagonalize an $N \times N$ matrix. Thus the present methods are particularly relevant to the orbital-free density functional methods 11, 12], which deal only with local pseudopotentials and can achieve $O(N)$ scaling for the entire calculation.

The Smooth Particle Mesh Ewald Method [1] (SPME) is an efficient scheme $(O(N \log N))$ for computing the Ewald energy $E^{\text {Ewald }}$ and its derivatives with respect to the atomic coordinates, $\partial E^{E w a l d} / \partial \mathbf{t}_{p}$. Here we show how similar ideas can be used to yield efficient methods (also $O(N \log N)$ ) for determining the ionic potential $V^{i o n}(\mathbf{r})$, and the other component of the BornOppenheimer forces, $\partial E^{e-i} / \partial \mathbf{t}_{p}$. 
We begin presentation of our method by expressing $V^{i o n}(\mathbf{r})$ in terms of the structure factor, $S(\mathbf{r})$ :

$$
V^{i o n}(\mathbf{r})=\frac{1}{\Omega} \int V^{p s p}\left(\mathbf{r}-\mathbf{r}^{\prime}\right) S\left(\mathbf{r}^{\prime}\right) d \mathbf{r}^{\prime}
$$

where

$$
S(\mathbf{r})=\Omega \sum_{\mathbf{R}} \sum_{p=1}^{N_{a t}} \delta\left(\mathbf{r}-\mathbf{t}_{p}-\mathbf{R}\right)
$$

and $\Omega=\left|\mathbf{a}_{1} \cdot\left(\mathbf{a}_{2} \times \mathbf{a}_{3}\right)\right|$ is the unit cell volume.

In reciprocal space, the expression for $V^{i o n}$ becomes:

$$
\begin{aligned}
\tilde{V}_{\mathbf{Q}}^{i o n} & \equiv \frac{1}{\Omega} \int_{\text {cell }} V^{i o n}(\mathbf{r}) e^{i \mathbf{Q} \cdot \mathbf{r}} d \mathbf{r} \\
& =\frac{1}{\Omega} \tilde{V}^{p s p}(\mathbf{Q}) \tilde{S}_{\mathbf{Q}}
\end{aligned}
$$

where

$$
\tilde{V}^{p s p}(\mathbf{Q}) \equiv \int V^{p s p}(\mathbf{r}) e^{i \mathbf{Q} \cdot \mathbf{r}} d \mathbf{r}
$$

and, using Eq. (5),

$$
\begin{aligned}
\tilde{S}_{\mathbf{Q}} & \equiv \frac{1}{\Omega} \int_{c e l l} S(\mathbf{r}) e^{i \mathbf{Q} \cdot \mathbf{r}} d \mathbf{r} \\
& =\sum_{p=1}^{N_{a t}} e^{i \mathbf{Q} \cdot \mathbf{t}_{p}}
\end{aligned}
$$

Inversely, we have:

$$
V^{i o n}(\mathbf{r})=\sum_{\mathbf{Q}} \tilde{V}_{\mathbf{Q}}^{i o n} e^{-i \mathbf{Q} \cdot \mathbf{r}}
$$

where the $\mathbf{Q}$-sum ranges over all integer multiples of the reciprocal lattice vectors $\mathbf{b}_{i}$ defined by $\mathbf{a}_{i} \cdot \mathbf{b}_{j}=2 \pi \delta_{i j}$.

For the purposes of the present work, we assume that $\rho(\mathbf{r})$ is represented on a $N_{1} \times N_{2} \times N_{3}$ grid of points $\mathbf{r}_{l_{1} l_{2} l_{3}}=\frac{l_{1}}{N_{1}} \mathbf{a}_{1}+\frac{l_{2}}{N_{2}} \mathbf{a}_{2}+\frac{l_{3}}{N_{3}} \mathbf{a}_{3}$, with $l_{i}=0, \ldots, N_{i}-1$. We will denote quantities such as $\rho\left(\mathbf{r}_{l_{1} l_{2} l_{3}}\right)$ as $\rho\left(l_{1}, l_{2}, l_{3}\right)$. The Fourier transform of $\rho(\mathbf{r})$ is approximated by:

$$
\tilde{\rho}\left(m_{1} \mathbf{b}_{1}+m_{2} \mathbf{b}_{2}+m_{3} \mathbf{b}_{3}\right) \simeq \mathcal{F}\left[\rho\left(l_{1}, l_{2}, l_{3}\right)\right]
$$

where $\mathcal{F}$ is the discrete Fourier transform:

$$
\begin{aligned}
\mathcal{F}\left[f\left(l_{1}, l_{2}, l_{3}\right)\right] & =\frac{1}{N} \sum_{l_{1}=0}^{N_{1}-1} \sum_{l_{2}=0}^{N_{2}-1} \sum_{l_{3}=0}^{N_{3}-1} f\left(l_{1}, l_{2}, l_{3}\right) \\
& \times e^{2 \pi i\left(\frac{l_{1} m_{1}}{N_{1}}+\frac{l_{2} m_{2}}{N_{2}}+\frac{l_{3} m_{3}}{N_{3}}\right)}
\end{aligned}
$$

where $N \equiv N_{1} N_{2} N_{3}$. Its inverse, $\mathcal{F}^{-1}$, is given by:

$$
\begin{aligned}
\mathcal{F}^{-1}\left[\tilde{f}\left(m_{1}, m_{2}, m_{3}\right)\right] & =\sum_{m_{1}=0}^{N_{1}-1} \sum_{m_{2}=0}^{N_{2}-1} \sum_{m_{3}=0}^{N_{3}-1} \tilde{f}\left(m_{1}, m_{2}, m_{3}\right) \\
& \times e^{-2 \pi i\left(\frac{l_{1} m_{1}}{N_{1}}+\frac{l_{2} m_{2}}{N_{2}}+\frac{l_{3} m_{3}}{N_{3}}\right)}(12)
\end{aligned}
$$

Using Eq. (6), and transforming back to the real space grid, we can determine $V^{i o n}(\mathbf{r})$ via:

$$
\begin{aligned}
& V^{\text {ion }}\left(l_{1}, l_{2}, l_{3}\right)= \\
& \quad \frac{1}{\Omega} \mathcal{F}^{-1}\left[\tilde{P}\left(m_{1}, m_{2}, m_{3}\right) \tilde{S}\left(m_{1}, m_{2}, m_{3}\right)\right]
\end{aligned}
$$

where $\tilde{P}\left(m_{1}, m_{2}, m_{3}\right)$ is the array given by:

$$
\tilde{P}\left(m_{1}, m_{2}, m_{3}\right) \equiv \tilde{V}^{p s p}\left(m_{1}^{\prime} \mathbf{b}_{1}+m_{2}^{\prime} \mathbf{b}_{2}+m_{3}^{\prime} \mathbf{b}_{3}\right)
$$

and $m_{i}^{\prime}=m_{i}$ for $0 \leq m_{i} \leq N_{i} / 2$ and $m_{i}^{\prime}=m_{i}-N_{i}$ otherwise. The array $\tilde{S}\left(m_{1}, m_{2}, m_{3}\right)$ is given by

$$
\tilde{S}\left(m_{1}, m_{2}, m_{3}\right) \equiv \tilde{S}\left(m_{1} \mathbf{b}_{1}+m_{2} \mathbf{b}_{2}+m_{3} \mathbf{b}_{3}\right)
$$

Calculation of the structure factor via Eq. (8) will scale with the square of the system size, because it needs to be computed at every reciprocal space grid point $\left(m_{1}, m_{2}, m_{3}\right)$, and at each of these points a sum must be performed over each atom in the system. However, Essmann et al., as part of their efficient Smooth Particle Mesh Ewald method[1], provide an elegant method for computing the structure factor efficiently (albeit approximately), requiring an amount of computation that scales as only $N \log N$. By incorporating this algorithm to compute the structure factor, the present methods for computing both the ionic potential $V^{i o n}(\mathbf{r})$ and the electron-ion contribution to the forces $\mathbf{F}_{p}^{e-i}$ achieve the same $O(N \log N)$ quasi-linear scaling.

Here we summarize the method of Essmann et al. for efficiently computing the structure factor, but refer the reader to the original reference 1] for full details. The crux of the algorithm lies in the approximation of the exponential in Eq. (8) with (complex) cardinal B-splines. The $n$th order cardinal B-spline function, $M_{n}(x)$, is defined as follows: $M_{2}(x)=1-|x-1|$ for $0 \leq x<2$, and $M_{2}(x)=0$ otherwise; and the higher-order cardinal B-splines are defined recursively:

$$
M_{n}(x)=\frac{x}{n-1} M_{n-1}(x)+\frac{n-x}{n-1} M_{n-1}(x-1)
$$

We define the grid coordinates of the $p$ th atom as $u_{i p} \equiv$ $N_{i} \mathbf{t}_{p} \cdot \mathbf{b}_{i}$, or equivalently $\mathbf{t}_{p}=\frac{u_{1 p}}{N_{1}} \mathbf{a}_{1}+\frac{u_{2 p}}{N_{2}} \mathbf{a}_{2}+\frac{u_{3 p}}{N_{3}} \mathbf{a}_{3}$. The structure factor, expressed in terms of the grid coordinates, is:

$$
\begin{aligned}
& \tilde{S}\left(m_{1}, m_{2}, m_{3}\right)=\sum_{p=1}^{N_{a t}} \exp \left(2 \pi i \frac{m_{1}}{N_{1}} u_{1 p}\right) \\
& \quad \times \exp \left(2 \pi i \frac{m_{2}}{N_{2}} u_{2 p}\right) \exp \left(2 \pi i \frac{m_{3}}{N_{3}} u_{3 p}\right)
\end{aligned}
$$

The exponentials can be approximated by $n$th order cardinal B-splines, where $n$ is even, as:

$$
\begin{aligned}
\exp \left(2 \pi i \frac{m_{j}}{N_{j}} u_{j p}\right) \simeq b_{j}\left(m_{j}\right) & \sum_{k=-\infty}^{\infty} M_{n}\left(u_{j p}-k\right) \\
& \times \exp \left(2 \pi i \frac{m_{j}}{N_{j}} k\right)
\end{aligned}
$$


where $b_{j}\left(m_{j}\right)$ is:

$$
b_{j}\left(m_{j}\right)=\frac{\exp \left(2 \pi i(n-1) m_{j} / N_{j}\right)}{\left[\sum_{k=0}^{n-2} M_{n}(k+1) \exp \left(2 \pi i \frac{m_{j}}{N_{j}} k\right)\right]}
$$

When this B-spline approximation of the exponential is used in Eq. (17), it becomes a discrete Fourier transform:

$$
\begin{aligned}
& \tilde{S}\left(m_{1}, m_{2}, m_{3}\right) \simeq \tilde{B}\left(m_{1}, m_{2}, m_{3}\right) \\
& \quad \times \sum_{p=1}^{N_{a t}} \sum_{k_{1}, k_{2}, k_{3}=-\infty}^{\infty} M_{n}\left(u_{1 p}-k_{1}\right) M_{n}\left(u_{2 p}-k_{2}\right) \\
& \quad \times M_{n}\left(u_{3 p}-k_{3}\right) e^{2 \pi i\left(\frac{m_{1} k_{1}}{N_{1}}+\frac{m_{2} k_{2}}{N_{2}}+\frac{m_{3} k_{3}}{N_{3}}\right)} \\
& \quad=N \tilde{B}\left(m_{1}, m_{2}, m_{3}\right) \mathcal{F}\left[Q\left(l_{1}, l_{2}, l_{3}\right)\right]
\end{aligned}
$$

where

$$
\tilde{B}\left(m_{1}, m_{2}, m_{3}\right) \equiv b_{1}\left(m_{1}\right) b_{2}\left(m_{2}\right) b_{3}\left(m_{3}\right)
$$

and:

$$
\begin{array}{r}
Q\left(l_{1}, l_{2}, l_{3}\right)=\sum_{p=1}^{N_{a t}} \sum_{c_{1}, c_{2}, c_{3}=-\infty}^{\infty} M_{n}\left(u_{1 p}-l_{1}-c_{1} N_{1}\right) \\
\times M_{n}\left(u_{2 p}-l_{2}-c_{2} N_{2}\right) M_{n}\left(u_{3 p}-l_{3}-c_{3} N_{3}\right)
\end{array}
$$

The array $Q\left(l_{1}, l_{2}, l_{3}\right)$ can be computed quickly $\left(O\left(N^{a t}\right)\right)$, because it is only non-zero for sub-cubes of dimension $n \times n \times n$ located near each atom. It is because $\mathcal{F}[Q]$ can be computed with the fast Fourier transform (FFT) (which is performed in $O(N \log N)$ operations) that the structure factor itself can be computed with $O(N \log N)$ operations.

It is now easily seen how the structure factor algorithm provided by the SPME method can be used to yield an efficient method for computing $V^{i o n}\left(l_{1}, l_{2}, l_{3}\right)$. By substituting Eq. (20) into Eq. (13), we obtain:

$$
\begin{aligned}
V^{i o n}\left(l_{1}, l_{2}, l_{3}\right)=\frac{N}{\Omega} \mathcal{F}^{-1}[ & \tilde{P}\left(m_{1}, m_{2}, m_{3}\right) \tilde{B}\left(m_{1}, m_{2}, m_{3}\right) \\
& \left.\times \mathcal{F}\left[Q\left(l_{1}, l_{2}, l_{3}\right)\right]\right]
\end{aligned}
$$

In practice, Eq. (23) could be used to efficiently compute $V^{i o n}\left(l_{1}, l_{2}, l_{3}\right)$ with the following algorithm. First $Q\left(l_{1}, l_{2}, l_{3}\right)$ is computed via Eq. (22). Then $\mathcal{F}[Q]$ is obtained via the FFT. $\mathcal{F}[Q]$ is then multiplied by $\tilde{B}$ and $\tilde{P}$, defined by Eqs. (21) and (14). Then the inverse FFT of this product is computed and multiplied by $N / \Omega$, yielding the array $V^{i o n}\left(l_{1}, l_{2}, l_{3}\right)$.

The calculation of the electron-ion contribution to the ionic force, $\partial E^{e-i} / \partial \mathbf{t}_{p}$ can also be made efficient, and again this comes from expressing $\partial E^{e-i} / \partial \mathbf{t}_{p}$ in terms of the structure factor. In the discrete variable representation, the expression for the pseudopotential energy, Eq. (2), becomes:

$$
E^{e-i} \simeq \frac{\Omega}{N} \sum_{l_{1}, l_{2}, l_{3}} \rho\left(l_{1}, l_{2}, l_{3}\right) V^{i o n}\left(l_{1}, l_{2}, l_{3}\right)
$$

which can be viewed as a dot product of $\rho$ and $V^{i o n}$; we can also evaluate this dot product in reciprocal space, and taking into account that $\rho$ is an array of real numbers, the expression becomes:

$$
E^{e-i} \simeq \Omega \sum_{m_{1}, m_{2}, m_{3}} \mathcal{F}\left[\rho\left(l_{1}, l_{2}, l_{3}\right)\right]^{*} \mathcal{F}\left[V^{i o n}\left(l_{1}, l_{2}, l_{3}\right)\right](25)
$$

then, using Eq. (23) for $V^{i o n}$, this becomes:

$$
\begin{aligned}
E^{e-i} \simeq & N \sum_{\substack{m_{1}, m_{2}, m_{3}\\
}} \mathcal{F}\left[\rho\left(l_{1}, l_{2}, l_{3}\right)\right]^{*} \tilde{P}\left(m_{1}, m_{2}, m_{3}\right) \\
& \times \tilde{B}\left(m_{1}, m_{2}, m_{3}\right) \mathcal{F}\left[Q\left(l_{1}, l_{2}, l_{3}\right)\right]
\end{aligned}
$$

Following this substitution, we now express the dot product in real space again:

$$
\begin{aligned}
E^{e-i} \simeq & \sum_{l_{1}, l_{2}, l_{3}} Q\left(l_{1}, l_{2}, l_{3}\right) \mathcal{F}^{-1}\left[\mathcal{F}\left[\rho\left(l_{1}, l_{2}, l_{3}\right)\right]\right. \\
& \left.\times \tilde{P}\left(m_{1}, m_{2}, m_{3}\right)^{*} \tilde{B}\left(m_{1}, m_{2}, m_{3}\right)^{*}\right]
\end{aligned}
$$

Because the only factor that depends on the atomic positions is $Q$, we can readily differentiate with respect to the atomic positions:

$$
\begin{aligned}
\frac{\partial E^{e-i}}{\partial \mathbf{t}_{p \alpha}}= & \sum_{l_{1}, l_{2}, l_{3}} \frac{\partial Q}{\partial \mathbf{t}_{p \alpha}} \mathcal{F}^{-1}\left[\mathcal{F}\left[\rho\left(l_{1}, l_{2}, l_{3}\right)\right]\right. \\
& \left.\times \tilde{P}\left(m_{1}, m_{2}, m_{3}\right)^{*} \tilde{B}\left(m_{1}, m_{2}, m_{3}\right)^{*}\right]
\end{aligned}
$$

where $\alpha=1,2,3$ is the vector component of the force. Eqs. (23) and (28) (and the expression for the stress tensor, Eq. (A3) constitute the central results of this article.

The partial derivatives $\partial Q / \partial \mathbf{t}_{p \alpha}$ can be evaluated readily with the definition of $Q$, Eq. (22), and with the aid of the following B-spline identity:

$$
\frac{d}{d x} M_{n}(x)=M_{n-1}(x)-M_{n-1}(x-1)
$$

Computing the partial derivatives of Eq. (28) for all of the atoms in the system requires an amount of computation that scales as $N \log N$. As with $Q$, the partial derivative array $\partial Q / \partial \mathbf{t}_{p \alpha}\left(l_{1}, l_{2}, l_{3}\right)$ is only non-zero in sub-cubes near the atomic positions, so computing it requires $O(N)$ computation. The fast Fourier transforms scale as $N \log N$.

The application of Eq. (28) for rapid computation of the electron-ion forces $\mathbf{F}^{e-i}$ can proceed algorithmically as follows. The Fourier transform of $\rho\left(l_{1}, l_{2}, l_{3}\right)$ is computed; then $\mathcal{F}[\rho]$ is multiplied by $\tilde{P}^{*}$ and $\tilde{B}^{*}$. Then the inverse Fourier transform of this product is found. Then by utilizing Eq. (29), the derivatives $\partial Q / \partial \mathbf{t}_{p \alpha}\left(l_{1}, l_{2}, l_{3}\right)$ are computed during the summing over the $l_{i}$ 's, yielding $\partial E^{e-i} / \partial \mathbf{t}_{p \alpha}$.

Similar ideas can yield an efficient expression for the computation of the stress tensor. The details of the efficient stress tensor method are covered in the appendix. 
Although these methods have been presented assuming that only one type of pseudopotential (and hence only one type of ion) is present in the system, multiple types are readily treated. Since the ionic potential is a linear function of the pseudopotentials, then with multiple types of pseudopotentials the total ionic potential is a sum of ionic potentials of the different types,

$$
V_{\text {tot }}^{\text {ion }}\left(l_{1}, l_{2}, l_{3}\right)=\sum_{\tau} V_{\tau}^{\text {ion }}\left(l_{1}, l_{2}, l_{3}\right)
$$

where $\tau$ indexes the pseudopotential type. The individual $V_{\tau}^{\text {ion }}$ are each computed with Eq. (23) using the $Q$-array associated with this set of ions, and the $\tilde{P}$-array associated with this pseudopotential type. Likewise to compute the ionic forces, Eq. (28) is used to compute the forces on all ions of a given pseudopotential type, using the $Q$-array associated with this set of ions, and the $\tilde{P}$-array associated with this pseudopotential type.

Several tests have been performed to examine the accuracy of these methods. The accuracy of the approximate structure factor, Eq. (20), increases when the number of grid points $N$ increases, and when the B-spline order $n$ is increased. However, in an electronic structure calculation, one is not simply free to choose the number of grid points for computing $\tilde{S}$. It is clear from Eqs. (23) and (28) that $\tilde{S}$ and the electronic charge density $\rho$ must exist on the same grid, and typically energy convergence considerations dictate a minimum grid density on which $\rho$ is represented. So it must be established that for a given grid density, the error incurred by using the approximate $\tilde{S}$ when generating $V^{i o n}$ and calculating the forces (i.e. the present methods, Eqs. (23) and (28)) is not much more than the error that would be present using the same finite number of grid points and the exact structure factor $S$ when generating $V^{i o n}$ and calculating the forces. In other words, it must be shown that the error in the total energy and forces that comes from using an approximate $\tilde{S}$ is smaller than the error due to using a grid of finite density. Furthermore, in order to establish that these methods are indeed (quasi)linear scaling, it must be demonstrated that for a fixed grid density $N / \Omega$ and fixed B-spline order $n$, the error per atom due to the approximate $\tilde{S}$ does not increase when the system size is increased.

All tests here have been done on systems of aluminum atoms simulated with orbital-free density functional theory 11, 12]. The present methods for generating $V^{i o n}\left(\mathbf{r}_{i}\right)$ (i.e. the method of Eq. (23)) and $\mathbf{F}^{e^{-i}}$ (Eq. (28)) were tested as follows: first, in order to test the error due to the approximate $\tilde{S}$ compared to the error from the rest of the calculation, $32 \mathrm{Al}$ atoms were placed in a cubic box $8.08 \AA$ on a side, and displaced randomly by about $0.5 \AA$ from their fcc crystalline positions. Then $V^{i o n}$ was generated using the exact $\tilde{S}$ (Eq. (8) ), and the present method (Eq. (23)) with B-spline orders $n=6,8,10,12$, and separate electronic relaxations were done in each of these $V^{i o n}$ 's, yielding corresponding total energies. After electronic relaxation, electron-ion forces

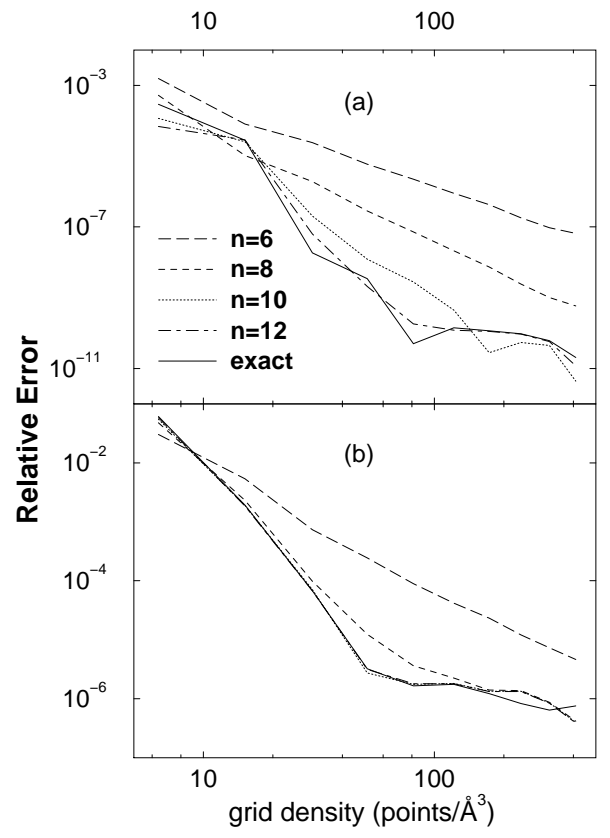

FIG. 1: (a) Relative error in the energy compared to infinite grid density limit, Eq. (31). (b) Relative error in $\mathbf{F}^{e-i}$ compared to the infinite grid limit, Eq. (32).

$\mathbf{F}^{e-i}$ were calculated using derivatives of the exact $\tilde{S}$, and with the present method (Eq. (28)). This was done for successively higher grid densities. The results are shown in Fig. 1 The relative error between the energy and the converged energy, as measured by:

$$
\frac{\left|E-E^{\infty}\right|}{\left|E^{\infty}\right|}
$$

(where $E^{\infty}$ is the energy in the limit of infinite grid density) is plotted as a function of grid density for different choices of $V^{i o n}: V^{i o n}$ generated with the exact method and the present method. $E^{\infty}$ is taken to be the total energy evaluated with a grid density of 800 points $/ \AA^{3}$, a considerably higher density than plotted in Fig. 1 thus the deviation from this grid's energy from the true $E^{\infty}$ is of a smaller order of magnitude than the energy deviations found at the grid densities explored in Fig. 1] making it a suitable energy to use as $E^{\infty}$. It is clear that with a B-spline order of $n=10$ the error due to the use of the approximate $V^{i o n}$ is negligible compared to the error due to the finite grid density. Also plotted is the relative error in the calculated electron-ion forces $\mathbf{F}^{e-i}$. The method used for calculating the force corresponded to the method used to calculate $V^{i o n}$; e.g. the data for the forces calculated with the present method and a 6th order B-spline were done with charge densities relaxed in a $V^{i o n}$ generated with the present method with a 6 th order B-spline. Thus errors in the forces that were calculated with the present method have some error contribution from using the present method for generating $V^{i o n}$. The relative force error was measured as the fractional root- 


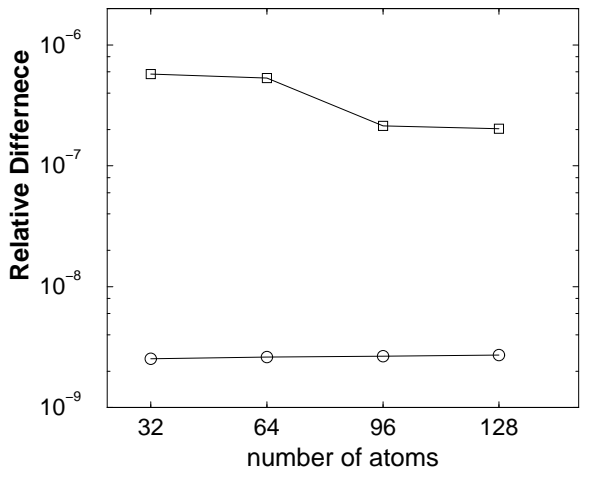

FIG. 2: The relative difference between the total energy (circles) and forces (squares) calculated with the present methods and with the exact structure factor methods, as a function of system size (see Eqs. (31) and (32).

mean-square deviation of each force component on each atom:

$$
\left[\frac{\sum_{i=1}^{N_{a t}} \sum_{\alpha}\left(F_{i \alpha}-F_{i \alpha}^{\infty}\right)^{2}}{\sum_{i=1}^{N_{a t}} \sum_{\alpha}\left(F_{i \alpha}^{\infty}\right)^{2}}\right]^{1 / 2}
$$

where the $\mathbf{F}_{i}^{\infty}$ 's are the forces in the limit of infinite grid density, in the same sense as expained above for $E^{\infty}$. In this case, already at a B-spline order of $n=8$ the forces are almost as accurate as those calculated with the exact method, and for $n=10,12$ the forces are indistinguishable.

Second, in order to verify the scaling of these methods for different system sizes, four different systems were considered. For systems of 32, 64, 96, and $128 \mathrm{Al}$ atoms, displaced from crystalline positions as before, $V^{\text {ion }}$ was generated with the exact $\tilde{S}$, and with an approximate $\widetilde{S}$ generated with 8 th-order B-splines. The grid density in each case was 237 points $/ \AA^{3}$. After electronic relaxation, forces were calculated using the exact $\tilde{S}$ or with the present method, again corresponding to the method used to generate $V^{i o n}$. The relative error between these energies and forces as a function of system size is plotted in Fig. 2. The relative errors are measured as before, with expressions like Eqs. (31) and (32), but instead of comparing to the energy and forces of the infinite grid density limit, the energy and forces are compared to those calculated using the same grid density and the exact structure factor of Eq. (8). The relative error in the energy is seen to be constant as a function of system size, and the relative error in the forces is actually seen to decrease slightly with increasing system size. Thus sufficient accuracy can be achieved with the present methods for calculating $V^{i o n}$ and the $\mathbf{F}^{e-i}$ using a fixed B-spline order and grid density, confirming that these methods will scale quasi-linearly with system size.

Finally, the time required to generate $V^{i o n}$ with the exact and present methods, and to evaluate the electron-ion forces with the exact and the present methods is plotted

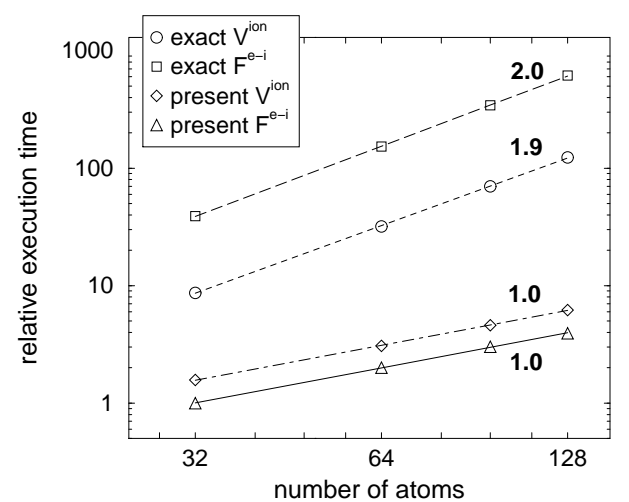

FIG. 3: The computation time required to generate $V^{i o n}$ and evaluate the $\mathbf{F}^{e^{-i}}$, s as a function of number of atoms, for the traditional and present methods. The numbers on each curve represent the exponent of a power law fit.

in Fig. [3] One unit of time in the figure corresponded to 1.38 seconds of execution time on a $450 \mathrm{MHz}$ Pentium III processor. The present methods are far superior even for modest numbers of atoms. It is also noted that the present methods are readily parallelized.

In conclusion, we present accurate and efficient methods for computing the ionic potential and the BornOppenheimer forces on atoms by utilizing an approximate form of the structure factor. It has been demonstrated that the errors due to the present methods do not increase with increasing system size. As is typically the case with approximate numerical methods, there is a trade-off between accuracy and efficiency with the present methods. Accuracy can be systematically improved by increasing the grid density or by increasing the B-spline order $n$, both at the expense of more computing time. However, it was demonstrated that the errors introduced by using the present methods at a B-spline order of $n=10$ are small compared to errors in other parts of the electronic structure calculation that arise due to the use of a finite grid density. Thus a B-spline order of 10 is recommended as the optimal compromise for simple metals like $\mathrm{Al}$, which was used here as a test case.

\section{APPENDIX A: THE ELECTRON-ION CONTRIBUTION TO THE STRESS TENSOR}

The present methods are readily applied to the computation of the electron-ion contribution to the stress tensor. The stress tensor can be calculated with the methods of Nielsen and Martin [15]. One constructs an ionic potential skewed by a strain tensor $\epsilon$, and a density similarly skewed and scaled to preserve normalization:

$$
\begin{aligned}
V_{\epsilon}^{i o n}(\mathbf{r}) & \equiv V^{i o n}\left((\mathbb{1}+\epsilon)^{-1} \mathbf{r}\right) \\
\rho_{\epsilon}(\mathbf{r}) & \equiv[\operatorname{det}(\mathbb{1}+\epsilon)]^{-1} \rho\left((\mathbb{1}+\epsilon)^{-1} \mathbf{r}\right)
\end{aligned}
$$


The electron-ion contribution to the stress density tensor is then given by:

$$
\Omega \sigma_{\alpha \beta}^{e-i}=\left.\frac{\partial E_{\epsilon}^{e-i}}{\partial \epsilon_{\alpha \beta}}\right|_{\epsilon \rightarrow \mathbf{0}}=\frac{\partial}{\partial \epsilon_{\alpha \beta}} \int_{\text {cell }_{\epsilon}} \rho_{\epsilon}(\mathbf{r}) V_{\epsilon}^{i o n}(\mathbf{r}) d \mathbf{r}(\mathrm{A} 2)
$$

sity, $\mathcal{F}\left[\rho\left(l_{1}, l_{2}, l_{3}\right)\right]$, are simply scaled by $[\operatorname{det}(\mathbb{1}+\epsilon)]^{-1}$. The reciprocal lattice vectors, to first order in $\epsilon$, become $\mathbf{b}_{i} \rightarrow(\mathbb{1}-\epsilon) \mathbf{b}_{i}$, and hence $\partial \mathbf{b}_{i \gamma} / \partial \epsilon_{\alpha \beta}=-\delta_{\alpha \gamma} \mathbf{b}_{i \beta}$. Differentiating Eq. (26) with respect to $\epsilon_{\alpha \beta}$, one obtains an efficient method for the electron-ion stress density tensor:

Under the strain transformation, the structure factor is unchanged, and the Fourier components of the den-

$$
\sigma_{\alpha \beta}^{e-i}=-\frac{E^{e-i}}{\Omega} \delta_{\alpha \beta}-\frac{N}{\Omega} \sum_{m_{1}, m_{2}, m_{3}} \frac{\mathbf{Q}_{\alpha} \mathbf{Q}_{\beta}}{|\mathbf{Q}|} \tilde{P}^{\prime}\left(m_{1}, m_{2}, m_{3}\right) \mathcal{F}\left[\rho\left(l_{1}, l_{2}, l_{3}\right)\right]^{*} \tilde{B}\left(m_{1}, m_{2}, m_{3}\right) \mathcal{F}\left[Q\left(l_{1}, l_{2}, l_{3}\right)\right]
$$

where $\mathbf{Q} \equiv m_{1}^{\prime} \mathbf{b}_{1}+m_{2}^{\prime} \mathbf{b}_{2}+m_{3}^{\prime} \mathbf{b}_{3}$, and $\tilde{P}^{\prime}\left(m_{1}, m_{2}, m_{3}\right) \equiv d \tilde{V}^{p s p}(\mathbf{Q}) / d|\mathbf{Q}|$. This method has been subjected to simple tests comparing the derivative of the energy with respect to the crystal lattice constant to the components of the stress tensor.
These tests indicate an accuracy similar to the present force method. It must be noted, however, that unlike the ionic forces, the cell stress has contributions from all terms in the energy, and $\sigma_{\alpha \beta}^{e-i}$ is merely one of them.
[1] U. Essmann, L. Perera, et al., J. Chem. Phys. 103 (19) 8577 (1995); T. Darden, D. York, and L. Pedersen, J. Chem. Phys. 9810089 (1993).

[2] W. Yang, Phys. Rev. Lett. 66, 1438 (1991).

[3] X.-P. Li, R. W. Nunes, and D. Vanderbilt, Phys. Rev. B 47, 10891 (1993); M. S. Daw, ibid. 47, 10895 (1993).

[4] P. Ordejón, D.A. Drabold, M.P. Grumbach, and R. M. Martin, Phys. Rev. B 4814646 (1993).

[5] W. Kohn, Phys. Rev. Lett. 763168 (1996); P. D. Haynes and M. C. Payne, Phys. Rev. B 59, 12173 (1999).

[6] J. Soler, E. Artacho, J. Gale, et al., J. Phys.: Cond. Mat. 14, 2745 (2002).

[7] S. Goedecker, Rev. Mod. Phys. 711085 (1999) and references therein.

[8] E. Hernańdez, M. J. Gillan, and C. M. Goringe, Phys.
Rev. B 537147 (1996).

[9] K. N. Kudin and G. E. Scuseria, Phys. Rev. B 6116440 (2000).

[10] Y. Shao, C. A. White, and M. Head-Gordon, J. Chem. Phys. 1146572 (2001).

[11] S. C. Watson and E. A. Carter, Comp. Phys. Commun.128 67 (2000).

[12] Y. A. Wang, N. Govind, and E. A. Carter, Phys. Rev. B 6016350 (1999).

[13] H. Hellmann, Einführung in die Quantenchemie (Deuticke, Leipzig, 1937), pp. 61 and 285.

[14] R. P. Feynman, Phys. Rev. 56340 (1939).

[15] O. H. Nielsen and R. M. Martin, Phys. Rev. B 323780 (1985); ibid. 323792 (1985). 